\title{
SUB-20 ps HIGH ENERGY PULSES FROM 1 kHz NEODYMIUM- BASED CPA
}

\author{
K. Michailovas ${ }^{\text {a, b }}$, A. Zaukevičius ${ }^{\text {a, c }}$, V. Petrauskienè a, V. Smilgevičius ${ }^{\text {b }}$, S. Balickas ${ }^{\text {a }}$, \\ and A. Michailovas ${ }^{\mathrm{a}, \mathrm{c}}$ \\ ${ }^{a}$ EKSPLA, Savanoriu 237, 02300 Vilnius, Lithuania \\ ${ }^{\mathrm{b}}$ Vilnius University Laser Research Center, Saulètekio 10, 10223 Vilnius, Lithuania \\ 'Institute of Physics, Center for Physical Sciences and Technology, Savanoriu 231, 02300 Vilnius, Lithuania \\ Email:k.michailovas@ekspla.com
}

Received 23 October 2017; accepted 21 June 2018

\begin{abstract}
Chirped pulse amplification (CPA) technique was realized in Nd:YVO and Nd:YAG amplifiers. The front-end of the system consists of an Yb-doped fiber-optic master oscillator and a chirped fiber Bragg grating stretcher with a chain of Nd-based solid-state amplifiers followed by a grating compressor with custom high-dispersion multilayer dielectric gratings. This allowed us to implement a relatively compact and moderately complex master oscillator power amplifier (MOPA) layout. $85 \mathrm{~mJ}$ of sub-20 ps pulses at $1 \mathrm{kHz}$ repetition rate was obtained at the output. The amplifier features favourable parameters for OPCPA pumping.
\end{abstract}

Keywords: chirped pulse amplification (CPA), neodymium amplifiers, picosecond pulse amplifier, Nd:YAG, $\mathrm{Nd}: \mathrm{YVO}_{4}$, optical parametric chirped pulse amplification (OPCPA)

PACS: 42.55.Xi, 42.60.By, 42.60.Da, 42.65.Re

\section{Introduction}

Extremely short laser pulses are achieved in optical parametric chirped pulse amplification (OPCPA) systems [1]. Such systems rely on amplification of ultrashort broadband signal pulses in nonlinear crystals pumped by a high-energy and high-intensity temporally synchronized pump laser. When chirping, the seed pulse spectrum is mapped onto a temporal pulse envelope. Thus, to preserve the pulse duration, the initial pulse spectrum must be preserved. From this point of view, an important aspect in high-energy OPCPA system design is the ratio of pump pulse and seed pulse durations. An optimal pump-to-seed pulse duration ratio exists which ensures a high energy conversion efficiency, simultaneously providing a stable temporal overlap of the broadband seed and pump pulses, as well as the overall stability of the system. Therefore, the pump pulse duration determines the required seed pulse stretching factor and the resulting amplification bandwidth. Generating short high-energy pulses for OPCPA pumping is a challenging task - a compromise between the pump pulse duration and the bandwidth of parametric amplification has to be achieved. The required high contrast of the amplified and compressed ultrashort pulses is another important challenge. The shorter the pump pulse, the easier it is to fulfill this requirement. Spectral phase control to ensure compression fidelity is yet another important aspect when generating 
few-optical-cycle pulses. For seed pulses stretched to a lesser extent, extremely short pulses can be achieved after compression with acousto-optic programmable dispersive filters (AOPDF) or similar devices.

OPCPA systems with Yb:YAG-based pump sources featuring sub-picosecond pulse duration are widely spread; yet, they must fulfill strict requirements on synchronization of pump and seed pulses, as well as overall system stability [2, 3]. Moreover, power scaling of Yb:YAG laser amplifiers is highly constrained and has a large impact on system complexity and price. Nd-based laser systems are much less complex. Nevertheless, due to a relatively narrow amplification bandwidth of Nd-doped active media, the supported pulse durations are at least several times longer than those achievable by, for example, Yb:YAG active media. Multi-millijoule Nd-based laser systems typically produce pulses in a range of $20-100 \mathrm{ps}$ [4, 5], while pulses below 20 ps are limited to small pulse energies and low average powers [6]. There is a great demand for $100 \mathrm{~W}$-level pump pulse sources with pulse duration in the 10-20 ps range for pumping of OPCPA systems.

Compression of the second harmonic pulses generated from chirped fundamental pulses is a method that allows achieving the shortest pulse durations from Nd-based chirped pulse amplifiers (CPA) [7]. It allows achieving pulse durations up to two times shorter after compression. The main limiting factor for this approach is the efficiency of the compressor gratings.

This work is a continuation of our efforts [ 8,9 , 9$]$ in development of a CPA concept using neodymium based amplifiers with the purpose of creating a moderately complex $100 \mathrm{~W}$-class pump source for OPCPA systems. In our previous work, we have observed a slight spectral broadening of chirped pulses after power amplification. This effect cannot be explained just by spectral broadening due to self-phase modulation. In [8, 9], we attempted explaining the effect qualitatively by spectral shifting during amplification in a power amplifier that was spectrally detuned from a regenerative amplifier. To find the cause of this spectral broadening effect, we have performed modelling of amplification in a Nd-based CPA. Another goal was to find the methods to control the broadening and to apply them in the Nd-based CPA with the goal of obtaining the shortest possible compressed pulse duration.

Here we present numerical modelling results and a Nd-based CPA system consisting of a chain of $\mathrm{Nd}: \mathrm{YVO}_{4}$ and Nd:YAG amplifiers with 32\% overall system optical-to-optical efficiency, which is important for the total system thermal management and build cost. We obtained $100 \mathrm{~mJ}$-level pulses while retaining the bandwidth of the initial spectrum of the seed source. The mechanism that allowed us to preserve the bandwidth of the spectrum was amplification of chirped pulses under gain-saturation conditions. By means of detuning the central wavelength of the seed radiation with respect to the center of the amplification band (or vice versa), this effect can be controlled and strengthened. The amplifier presented in this paper could be used as a pump source for a compact stand-alone OPCPA system.

\section{Numerical simulations}

Numerical simulations of gain-saturated chirped pulse amplification were performed in order to understand the impact that the initial pulse spectrum and amplification band have on the output radiation spectrum when operating in a gain-saturation mode.

The simulations were based on the Frantz-Nodvik theory [10] adapted for chirped pulses [11-13], simultaneously accounting for the wavelengthdependent gain profile [14]. None of the previous works [11-13] analysed CPA in narrowband active media. We performed the simulations (1D approach) for $\mathrm{Nd}$ :YAG and $\mathrm{Nd}: \mathrm{YVO}_{4}$.

In the model, the chirped input pulse was split into multiple square-shaped time slices and each temporal slice was associated with a single spectral component (a carrier frequency at the instant of time in the center of the slice). Based on this, the slice of the pulse was considered as monochromatic [13], so that simple rate equations [15] are valid. Also, the segment of the active medium that each temporal slice occupies can be considered as bearing a uniform population inversion distribution. Pulse duration, and, consequently, all separate slices, are significantly shorter than the excited state lifetime. Therefore, we could apply a steady-state solution for square shaped pulses, known as the Frantz-Nodvik equation [10, 15] 


$$
\begin{aligned}
& F_{\text {out }}(v, z)=F_{\text {sat }}(v) \\
& \times \ln \left\{1+G(v, z)\left[\exp \left(\frac{F_{\text {in }}(v, z)}{F_{\text {sat }}(v)}\right)-1\right]\right\}, \\
& G(v, z)=\exp (g(v, z) \Delta z) \\
& g(v, z)=\sigma_{\mathrm{em}}(v) \cdot N_{2}(z)
\end{aligned}
$$

where $F_{\text {in }}(v, z)$ and $F_{\text {out }}(v, z)$ are input and output fluences, respectively, of a particular time slice of the pulse at a selected segment of the medium. $F_{\text {sat }}(v)$ is the frequency-dependent saturation fluence of the medium, while $\sigma_{\mathrm{em}}(v)$ is the effective emission cross-section of a Lorentz profile assuming that a homogeneous broadening dominates. $G(v, z)$ and $g(v, z)$ are the small signal gain factor and the gain coefficient, respectively, which depend on the frequency of the temporal slice and longitudinal coordinate in the active medium (in the segment of the medium where the time slice is amplified). $\Delta z$ is the length of the active medium segment that one temporal slice occupies, $\mathrm{N}_{2}(z)$ is the population inversion density at a particular segment of the active medium.

The small signal gain coefficient for the next time slice becomes lowered because energy has been extracted from that segment of the medium [15]:

$$
g^{\prime}\left(v^{\prime}, z\right)=\left(1-\frac{F_{\text {out }}(v, z)-F_{\text {in }}(v, z)}{g(v, z) \Delta z \cdot F_{\text {sat }}(v)}\right) g(v, z) .
$$

The saturation fluence $F_{\text {sat }}(v)$ for a 4-level laser medium depends on the photon energy $\mathrm{h} v$ and the emission cross-section $\sigma_{\text {em }}(v)$ value for a certain spectral component as follows:

$$
F_{\text {sat }}(v)=\frac{\mathrm{h} v}{\sigma_{\mathrm{em}}(v)} .
$$

In $\mathrm{Nd}: \mathrm{YVO}_{4}$, it is easier to achieve gain saturation compared to Nd:YAG due to a higher emission cross-section. For convenience, the concept of saturation energy is used:

$$
E_{\text {sat }}(v)=A \times F_{\text {sat }}(v) .
$$

Here $A$ is the laser mode area. One may compare the input pulse energy $E_{\text {in }}$ and the stored energy $E_{\text {stored }}$ with the saturation energy to assess whether pulse amplification occurs at gain-saturation mode.
The concept of modified saturation fluence is described in literature [16]. It accounts for a relatively long terminal level lifetime of the active medium, which is important for amplification of picosecond pulses. The formalism with the Frantz-Nodvik equation is still applicable here. Similarly, we used the concept of effective saturation fluence $F_{\text {sat eff }}=B \times F_{\text {sat }}$ in our model, where $B$ is a fitting factor to match the empirically observed input and output parameters. Accordingly, the input pulse energy $E_{\text {in }}$ and the stored energy $E_{\text {stored }}$ are compared with the effective saturation energy $E_{\text {sat eff }}=A \times F_{\text {sat eff }}$ For example, in the pre-amplifier of our system (Fig. 2), the factor $B=0.2$ was suitable to match the observed input and output energies with other parameters fixed. We believe it accounts for the terminal level lifetime and uncertainties of the model, also the error in laser mode size measurement.

The modelling results in Fig. 1 present the variation of the output spectrum $\left(S_{\text {out }}\right)$ when the central wavelength of the amplification band was tuned from $1063.8 \mathrm{~nm}$ to $1065.4 \mathrm{~nm}$. For convenience, we will call amplifier central wavelength detuning towards shorter wavelengths from the seed pulse central wavelength blue-shifted amplifier, and detuning to longer wavelengths red-shifted amplifier. The input spectrum was fixed at $1064.6 \mathrm{~nm}$. A $0.8 \mathrm{~nm}$ FWHM of the amplification band was chosen while the input spectrum was $0.16 \mathrm{~nm}$ FWHM (similar to the input spectrum of the pre-amplifier (PreA) of our system (Fig. 2)). Input pulses were positively chirped. The simulation was carried out for the input pulse energy $E_{\text {in }} \approx 5 \times E_{\text {sat eff }}$ and the stored en$\operatorname{ergy} E_{\text {stored }} \approx 30 \times E_{\text {sat eff }}$ The initial population inversion density was assumed uniform along the active medium, which is true for laser diode side-pumped modules and is a very good approximation for laser diode double-end-pumped amplifier geometry.

The calculated output spectra $S_{\text {out }}$ differ in their shape and width for both the blue- and redshifted amplifier cases. The chosen $E_{\text {in }}$ and $E_{\text {stored }}$ values guarantee a gain-saturation mode for all amplification cases (red- and blue-shifted, and non-shifted). The width of the output spectrum strongly depends on the amplification band position with respect to the $S_{\text {in }}$ central wavelength: with no detuning, $S_{\text {out }}$ is slightly broader than $S_{\text {in' }}$, but the widest output bandwidth is obtained when the amplification band is red-shifted with respect to 

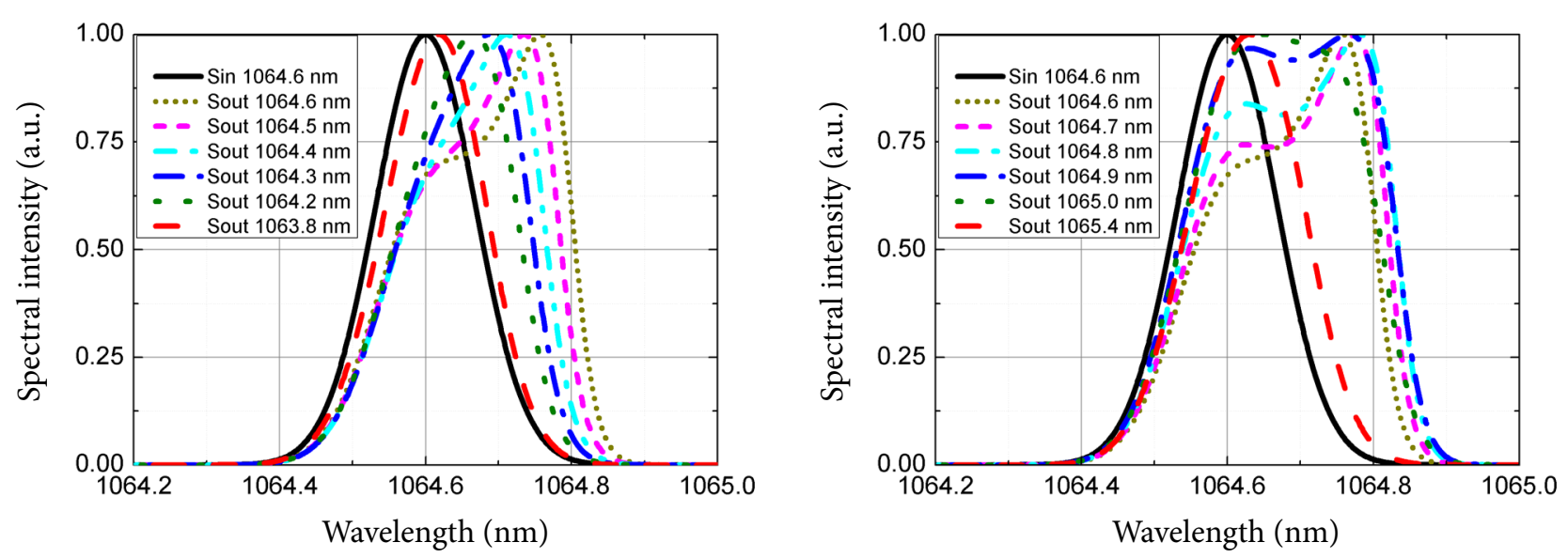

Fig. 1. Illustration of the numerical simulation results: simulated output spectra $\left(S_{\text {out }}\right)$ are shown for different central wavelengths of the amplification band (the numbers 1063.8-1065.4 $\mathrm{nm}$ indicate the central wavelength of the amplification band). The input spectrum $\left(S_{\text {in }}\right)$ was Gaussian with a fixed central wavelength of $1064.6 \mathrm{~nm}$ and bandwidth of $0.16 \mathrm{~nm}$ (FWHM). FWHM of the amplification band was $0.8 \mathrm{~nm}$. Other parameters: $E_{\text {in }} \approx 5 \times E_{\text {sat eff }} ; E_{\text {stored }} \approx 30 \times E_{\text {sat eff }}$

$S_{\text {in }}$. For the above-mentioned $E_{\text {in }}$ and $E_{\text {stored }}$ energies, the widest output bandwidth was obtained when the amplification band was red-shifted by about $0.3 \mathrm{~nm}$ (resulting in $S_{\text {out }}$ centered at $1064.9 \mathrm{~nm}$ ). The larger wavelength detuning produces a narrower spectrum again (that is still broader than the initial spectrum). As one can see, even for no detuning the spectrum broadens and experiences a red-shift during amplification. The red-shift can be compensated by a considerable detuning of the amplification band to the blue side in respect to the input radiation spectrum (the blue detuning of the amplification band), or detuning of the input radiation spectrum to the red side in respect to the amplification band. Broadening of the spectrum and changes of its shape are minimized under these conditions. Reducing the blue detuning of the amplification band increases the red-shift of the output spectrum and increases the broadening of the spectrum. Detuning the amplification band to the red side further

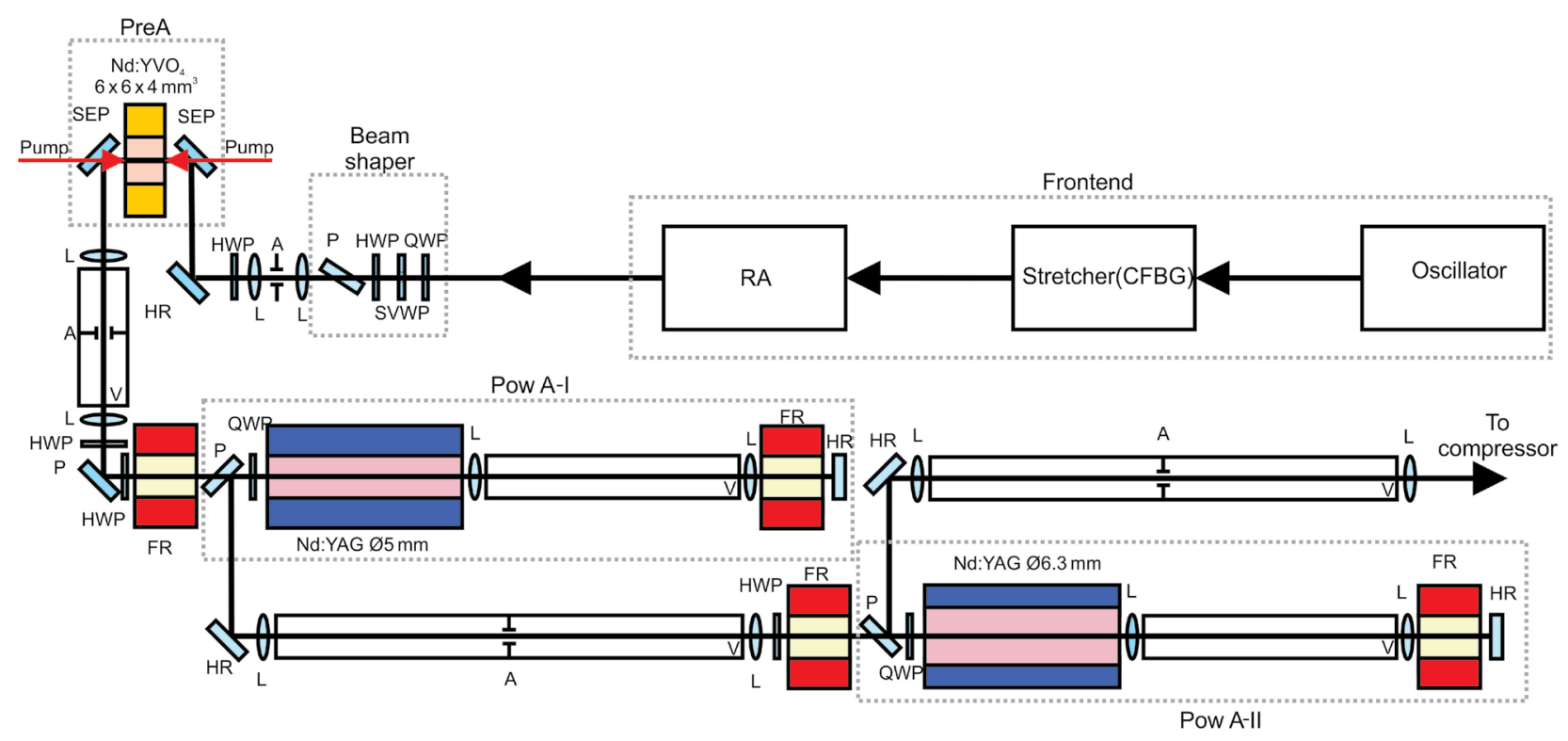

Fig. 2. A schematic layout of the amplification system. PreA, pre-amplifier; PowA-I and PowA-II, 1st and 2nd stages of power amplifier; RA, regenerative amplifier; CFBG, chirped fiber Bragg grating; QWP, quarter wave plate; HWP, half wave plate; SVWP, spatially variable wave plate; P, polarizer; L, lens; A, aperture; HR, high reflectivity mirror; SEP, $808 \mathrm{~nm} / 1064 \mathrm{~nm}$ separator; V, vacuum cell; FR, Faraday rotator. 
increases the spectral broadening until, at some point, both the broadening of the spectrum and the red-shift start to decrease. These transformations of the spectrum are governed by the fact that, in a positively chirped pulse, blue spectral components are delayed in time in respect to red spectral components. This means that the red spectral components are located at the front of the signal pulse and reach the amplifier earlier, when population inversion in amplifier medium is not affected yet. The earlier the spectral components reach the amplifier, the less depleted the population inversion is, and the higher the amplification they experience. This is the reason for the redshift in the amplified pulse spectrum. At the same time, other spectral components could experience a similar or even higher amplification because of being closer to the peak of the amplification band. Together with red-shifting, this results in spectral broadening. Therefore, our 1D simulations for saturated $\mathrm{Nd}: \mathrm{YAG}$ and $\mathrm{Nd}: \mathrm{YVO}_{4}$ active media showed - and qualitatively verified experimental results - that positively chirped pulses together with the red-shifting of the spectrum experience notable shape modifications.

Tuning of the spectral position of the amplification band may be achieved by exploiting its dependence on the temperature of the active medium [14] or by adjusting the wavelength of the seed pulse. It should be noted that tuning of the amplification band central wavelength demonstrated in Fig. 1 is extreme and would require temperature changes of the active medium in a range of $300^{\circ} \mathrm{C}$; this is just an illustration of the influence of spectral detuning on the spectral properties of the amplified pulse. The same result can be achieved easier using wavelength tuning of the input radiation, e.g. by means of selective filtering of certain wavelengths from a broadband seeder. A combination of both of these techniques could potentially give the best results. In our system, we tuned the central wavelength of the input radiation by heating the fiber oscillator's Bragg grating from 35 to $90^{\circ} \mathrm{C}$ (tuning range of about $0.4 \mathrm{~nm}$ ).

To sum up, gain-saturation in CPA has a strong impact on the output pulse spectrum. Among the governing parameters are ratios of the input pulse energy and the stored energy to the saturation energy, which in turn depends on the emission cross-section and the laser beam diameter.
Another important parameter is the wavelength detuning of incident radiation from the maximum of amplification transition of the active medium. Modelling showed that the seed radiation central wavelength detuning within a range of one amplifier bandwidth had a weak influence on the output energy and overall gain of the CPA, but gives a lot of freedom in adjusting the output spectrum. For properly chosen parameters, broadening of the amplified chirped pulse spectrum is achieved. Therefore, in contrast to the widespread opinion that amplifiers narrow the spectrum of amplified radiation, the saturated CPA may broaden it. This approach of wavelength detuning in CPA using Nd-doped active media was applied in designing the high-energy amplification system described below.

\section{System layout}

The amplification system (Fig. 2) started with a picojoule-level $\mathrm{Yb}$-doped fiber oscillator, with a thermally controlled Bragg grating as an end mirror for resonating radiation around $1064 \mathrm{~nm}$. Temperature control of the Bragg grating from 35 to $90^{\circ} \mathrm{C}$ could be used for tuning (within a $0.4 \mathrm{~nm}$ range) the central wavelength of the oscillator output radiation. The oscillator operated at $\sim 30 \mathrm{MHz}$ and produced $\sim 60 \mathrm{pJ}, 6 \mathrm{ps}$ pulses at $1064 \mathrm{~nm}$. The concept of the oscillator was the same as described in [9]. Oscillator output pulses were stretched up to 300 ps by a chirped fiber Bragg grating (CFBG). The CFBG was also placed into a heater to match its central wavelength to the central wavelength of the oscillator. Additionally, it was possible to create a linear gradient along the CFBG using this heater. This allowed controlling the chirp introduced by the stretcher and matching it to the dispersion of the compressor. The stretched pulses were amplified in a laser diode end-pumped $\mathrm{Nd}: \mathrm{YVO}_{4}$ regenerative amplifier (RA). The RA concept was similar to the regenerative amplifier described in [9], but with an additional laser diode pumping of the active medium from the opposite end (double-endpumping). The RA output pulses shortened to 150 ps due to gain-narrowing while the pulse energy was about $8 \mathrm{~mJ}$.

Using a spatially variable wave plate-based beam shaper [5], the Gaussian beam was converted to a flat-top beam described by the 14th order 
super-Gaussian function. The total transmission of the shaper was $50 \%$. A single-pass $6 \times 6 \times 4 \mathrm{~mm}$ $\mathrm{Nd}: \mathrm{YVO}_{4}$ laser diode end-pumped pre-amplifier (PreA) was used to double the pulse energy and compensate for the losses of beam shaping, providing an efficient seed for the Nd:YAG-based power amplifier.

In the two double-pass power amplification stages (PowA-I and PowA-II), laser diode side-pumped amplification Nd:YAG modules (Northrop Grumman Cutting Edge Optronics) with $\varnothing 5 \mathrm{~mm}$ and $\varnothing 6.3 \mathrm{~mm}$ rods were used. $130 \mathrm{~mJ}$ pulse energy at $1 \mathrm{kHz}$ repetition rate was achieved at the output of the power amplifier. Relay imaging between the active medium and the back reflecting mirror and a Faraday rotator were used in both stages to compensate for the thermally induced birefringence, with a quarter wave plate placed before each of the amplification modules to reduce the nonlinear interaction in the active media [17]. Relay imaging and spatial filtering were used between the amplification stages to preserve the shaped flat-top intensity distribution and suppress small scale self-focusing. The flat-top beam distribution allows one to efficiently utilize the population inversion accumulated in Nd:YAG rods, as a high fill factor [15] of about 0.7 can be reached without detrimental intensity distribution modulation caused by diffraction of the beam on the edge of the active medium.

Finally, the pulses were compressed using a twograting compressor (multilayer coated dielectric gratings manufactured by Fraunhofer IOF; 1818 lines $/ \mathrm{mm}, 150 \times 60 \mathrm{~mm}$ ) (Fig. 3). Gratings manufactured using this technology manifest supe- rior diffraction efficiency [18-20], which permits the total compressor transmission of over $90 \%$ and a high damage threshold in excess of $1 \mathrm{~J} / \mathrm{cm}^{2}$ [21]. The compressor was folded for compactness and used in a double-pass geometry with two bounces at both gratings (four bounces in total). A physical separation between the input and output beams was realized by a vertical periscope, comprised of two 45-degree mirrors to elevate the beam before the second pass through the compressor.

Several measures were taken to overcome beam profile degradation during the propagation over the long distance in the compressor. Firstly, we minimized the total length of the compressor. For this purpose, we limited pulse duration in the amplifier chain to 150 ps as compared to 300 ps in our previous work [9]. For this purpose a CFBG with dispersion parameter of $1000 \mathrm{ps} / \mathrm{nm}$ was used at the output of the master oscillator. The distance between the gratings of the compressor and the total optical path was reduced to $3 \mathrm{~m}$ (total) from $6 \mathrm{~m}$ in our previous work [9]. Secondly, the output beam of the amplification system was magnified about four times up to a diameter of over $20 \mathrm{~mm}$ in order to increase the distance the beam propagates without severe intensity modulation. The same magnifying telescope was used to image the beam from the output of the power amplifier to the symmetry plain of the compressor. The beam was then imaged from the symmetry plain of the compressor to the output of the system. This helped reduce detrimental diffraction effects on the output intensity distribution. These measures allowed us to preserve a flat-top-like intensity profile (Fig. 4) at

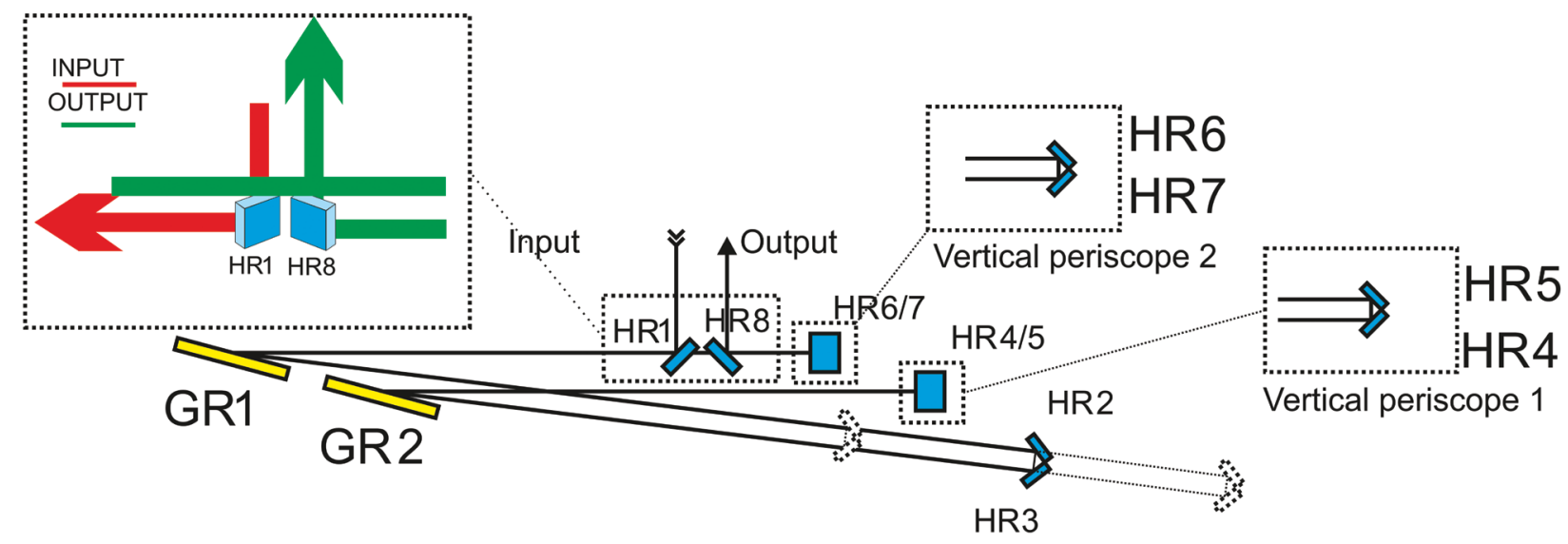

Fig. 3. Schematic layout of the compressor. GR1 and GR2 are diffraction gratings. Elements from HR1 to HR8 are high reflectivity mirrors (HR2 and HR3 are part of a delay line). 


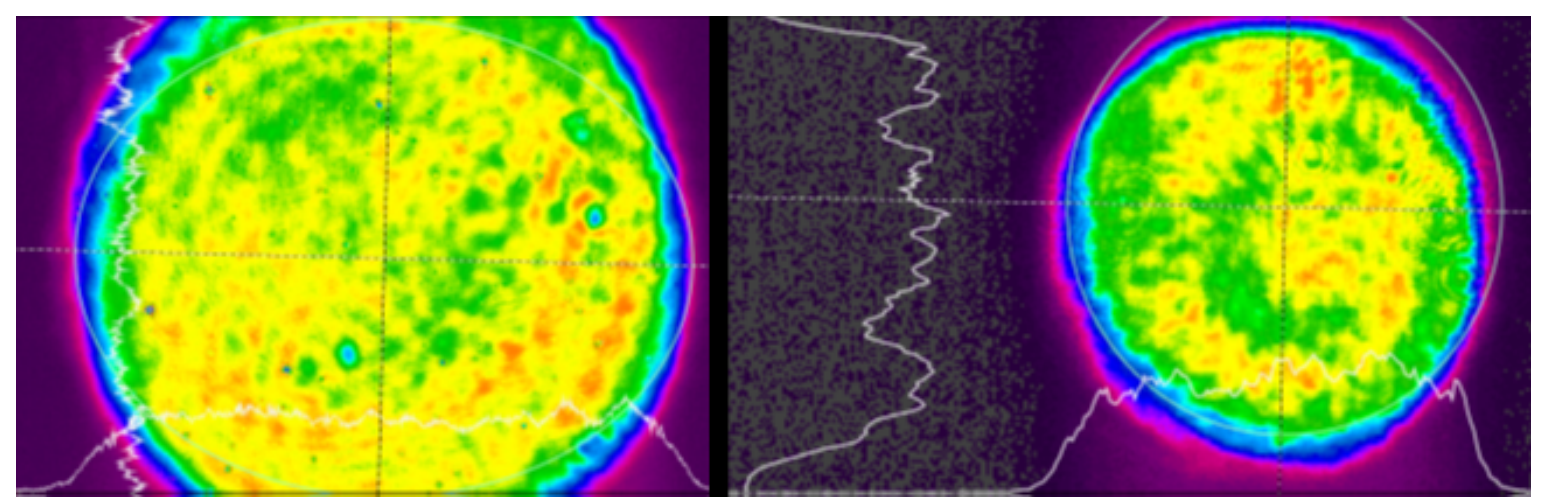

Fig. 4. Intensity distribution at the output of the amplification system (left) and at the output of the compressor (right).

the output of our system. The total throughput of the compressor was $66 \%$. This corresponds well with the diffraction efficiency values measured by the manufacturer: $95 \%$ average efficiency for one grating and $92 \%$ average efficiency for the other. The main factor in reducing the total efficiency of the compressor was a highly inhomogeneous reflectivity of the second grating, which varied from $96 \%$ to as low as $80 \%$.

\section{Results and discussion}

The main task of the CPA system design is to preserve spectral parameters of the initial radiation during amplification. This is done to avoid pulse shortening during amplification as it results in reduction of the safe limit of amplifier output energy. Another reason to preserve the broadest possible output spectrum is to achieve the shortest possible pulse durations after compression. We designed our system with this in mind. An Yb-doped fiber front-end was used, as it allows achieving sub-picosecond pulse durations in a relatively wide spectral range of 1020-1070 nm. Oscillator output pulses were stretched in time by a chirped fiber Bragg grating before being amplified in the subsequent regenerative amplifier (RA). It was the stage with the highest amplification factor in our system at $\sim 10^{5}$, thus manifesting the largest spectrum narrowing. We used an $\mathrm{Nd}-$ doped yttrium orthovanadate crystal $\left(\mathrm{Nd}: \mathrm{YVO}_{4}\right)$ in this stage of the system as it possesses several favourable features: a relatively large emission cross-section $\sigma_{\mathrm{em}}=114 \times 10^{-20} \mathrm{~cm}^{2}$ [22], which is about 4 times larger than that of Nd:YAG, and, more importantly, a broader amplification bandwidth. The two efficiency-limiting factors of the vanadate RA - a short fluorescence lifetime of about $90 \mu \mathrm{s}$ [22] and a long terminal level lifetime up to 870 ps [16] - were not critical to us.

The Nd:YVO ${ }_{4}$ pre-amplifier operated under a slight gain-saturation regime. After the pre-amplification, no spectral broadening was observed. This is due to the fact that it was not possible to adjust the temperatures of RA and PreA independently and to control the relative positions of central wavelengths of their amplification bands.

Finally, an Nd:YAG active medium was used in the power amplification stages because of its ability to store high energies and the availability of large active elements of a good optical quality. An amplification factor of about $2(130 \mathrm{~mJ} / 60 \mathrm{~mJ}=2.17)$ in the second power amplification stage PowA-II indicates a gain-saturation mode. The amplified pulse energies, amplified pulse bandwidths and spectral line shapes after every system stage are shown in Fig. 5. All spectra were measured by a Yokogawa 6373B spectrum analyzer.

We found that a slight mismatch of the amplification maxima of the final amplification stages (power amplifier) with the amplification maxima of RA (and fiber-optic seeder) and PreA allowed the bandwidth of the initial radiation to be preserved despite the narrowing effects in RA. The wavelength detuning in our system occurred due to differences in the operating temperatures of active media in different stages of the system, as well as the differences of spectral properties of the different active media used. The differences in temperature were mostly determined by geometry and average pump power, and could be controlled to some extent by changing the temperature of the coolant (water). Theoretically, 

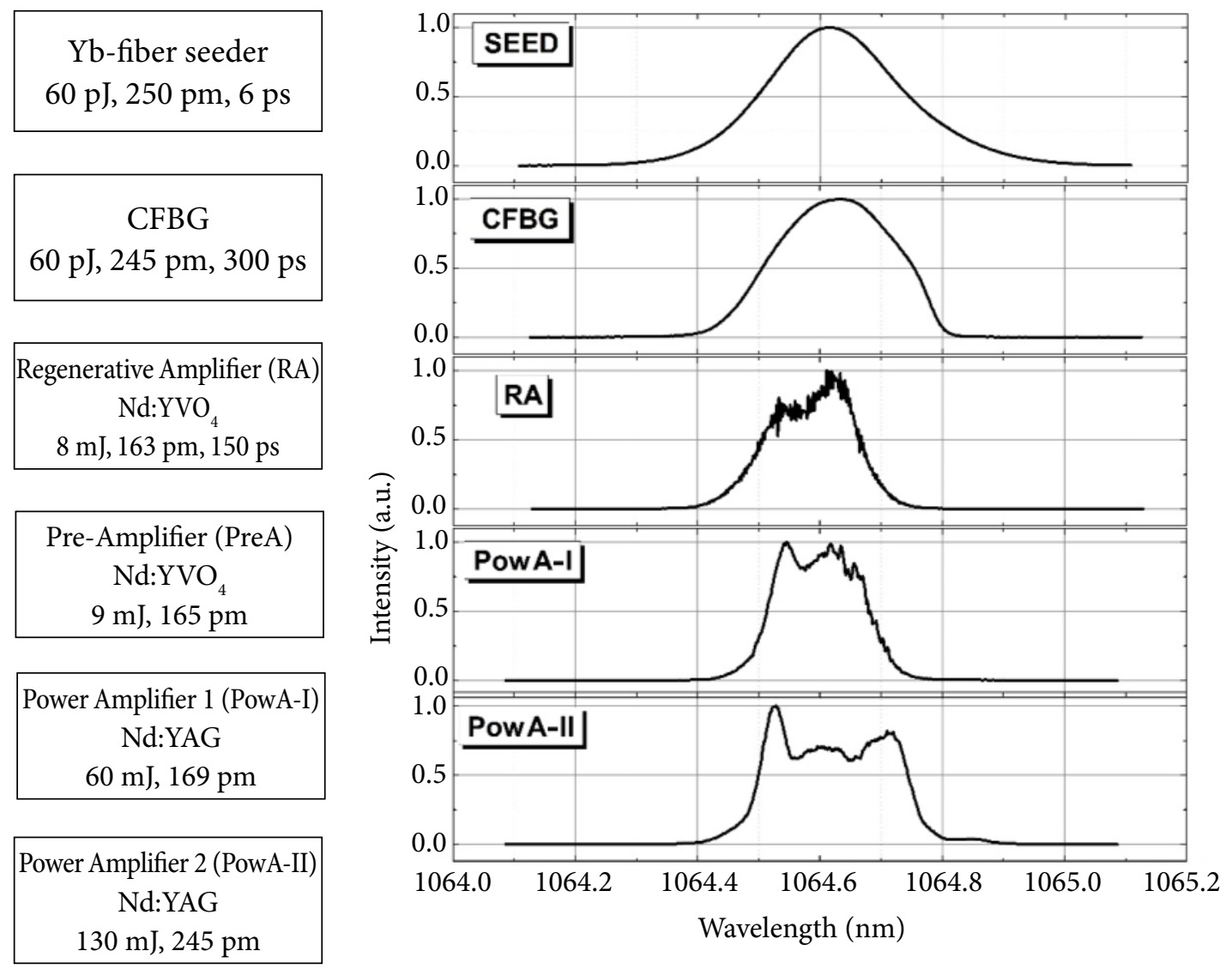

Fig. 5. Different parts of the amplification system with their parameters (left) and corresponding spectra (right).

one could shape the total amplification spectrum of the system by tuning the operating temperatures of separate components, but here we tuned the central wavelength of the seed only. We did this by changing the temperatures of the oscillator's FBG and the stretcher's CFBG.

The numerical simulations showed thas gainsaturated amplification has a strong impact on the spectrum of a chirped pulse. The wavelength detuning of incident radiation and the amplification spectrum is one of the main parameters to influence the resulting spectrum. The simple model in which the chirped pulse is sliced into quasimonochromatic portions revealed to be a good tool for a qualitative estimation of the importance of system parameters. It gave numerous hints for further system optimization. On the other hand, experimental data continuously contributed to development of the simulation code.

The measured spectra presented in Fig. 5 is a good illustration of the fact that a narrowband Nd-based amplification system is capable of retaining the spectrum of the initial broadband seed radiation incident to the amplification system. The spectra shown were measured for the case of no gradient on the stretcher CFBG, and the output spectrum is almost as broad (0.245 nm FWHM) as the output spectrum of the oscillator $(0.250 \mathrm{~nm}$ FWHM). Introducing a gradient (which increases the chirp of the stretcher) slightly narrows the output spectrum of the CFBG (to $0.188 \mathrm{~nm}$, for the largest gradient achievable by the used heater), which also narrows the output spectrum of the amplifier setup. A calculated bandwidth limited duration for the widest output spectrum achieved before compression was 8.3 ps. This was increased to 10.3 ps for the narrower spectrum achieved when a linear temperature gradient was created on the stretcher.

Pulse duration after compression was measured using a non-collinear second harmonics autocorrelator setup. The autocorrelation function (shown in Fig. 6) is noisy due to the measurement conditions: the beam wandering for our system was in the order of $150 \mu \mathrm{rad}$; the beam travelled a distance of several meters to the autocorellator 


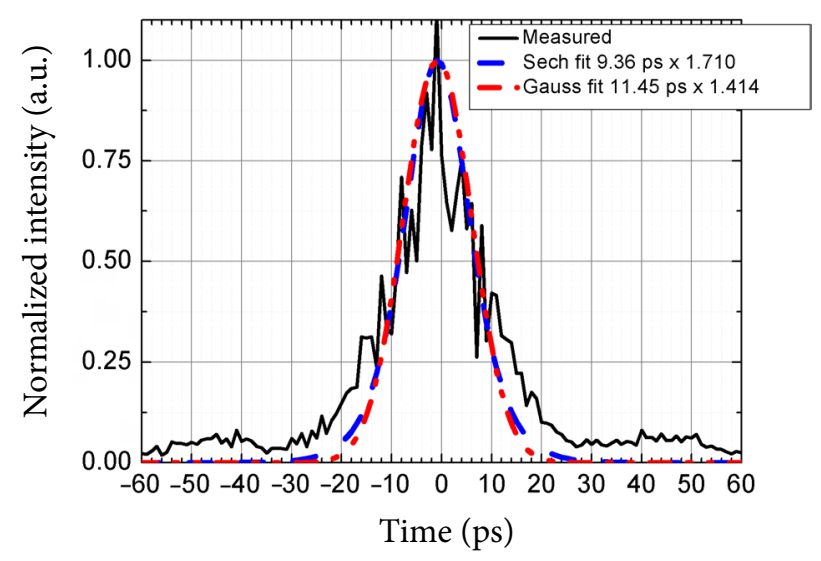

Fig. 6. Autocorrelation function after compression. The solid curve depicts measured values, the dashed curve shows the hyperbolic secant fit, and the dashdot curve is the Gaussian fit.

without imaging under a laminar air hood. The combination of beam wandering caused by the system itself and the effects of air movement on the beam could lead to shifting of the lateral position of the beam on the lens, focusing the beam into the second harmonic crystal, which in turn could lead to both lateral and longitudinal shifting of the position of the beam waist in the crystal, leading to a noisy autocorrelation measurement. The shortest pulses of $\sim 11.5$ ps (Fig. 6) were obtained with chirp control of the CFBG by changing the applied thermal gradient. Compression in the system was not optimal as we did not compensate for the third order group velocity dispersion. The measured autocorrelations for the minimal and maximal power had the same shape, indicating a negligible influence of the nonlinear phase modulation on the duration of the compressed pulse.

In our setup, we had no possibility to tune the PreA amplification band; nonetheless, we were able to preserve the amplified pulse spectrum compressible to the duration of the order of 10 ps. We see the potential in further development of our CPA system with the implementation of active media temperature control. For Nd-doped materials, to achieve a central wavelength shift of about $0.4 \mathrm{~nm}$, a change in temperature of about $100^{\circ} \mathrm{C}$ is needed. This is a theoretical evaluation based on wavelength dependence coefficients $4.3 \mathrm{pm} / \mathrm{deg}$ for $\mathrm{Nd}: \mathrm{YAG}$ and $3.4 \mathrm{pm} / \mathrm{deg}$ for $\mathrm{Nd}: \mathrm{YVO}_{4}$ crystals in a temperature range of $50-150^{\circ} \mathrm{C}$ [14]. A slightly larger wavelength tuning rate was obtained for $\mathrm{FBG}-7.1 \mathrm{pm} / \mathrm{deg}$ in a range of $30-95^{\circ} \mathrm{C}$. Employment of a seeder with a broader radiation spec- trum and independent control of temperature of all amplification stages could potentially result in even shorter pulses after compression, while a nonlinear gradient applied to the chirped fiber Bragg grating of the stretcher could improve the quality of the compression by compensating for the third order of dispersion.

\section{Summary}

In this work we developed a neodymium-based CPA system with an Yb-fiber seeder for OPCPA pumping, producing $85 \mathrm{~mJ}$ (130 mJ before compression) sub-20 ps pulses at $1 \mathrm{kHz}$ repetition rate $(85 \mathrm{~W}$ average power). The shaped flat-top-like beam profile was preserved throughout the entire system by incorporation of relay imaging. Most of the bandwidth (98\% at FWHM level) of the input pulse has been preserved by tuning the central wavelength of the seed radiation. The results of numerical simulation showed that for chirped pulse amplifier systems based on active media with dominating homogeneous broadening, bandwidth broadening of the amplified pulse could be achieved by properly choosing operational parameters.

\section{Acknowledgements}

We would like to thank Jonas Adamonis for the beam wandering measurements.

\section{References}

[1] S. Witte and K. Eikema, Ultrafast optical parametric chirped-pulse amplification, IEEE J. Sel. Top. Quantum Electron. 18, 296-307 (2012), https://doi.org/10.1109/JSTQE.2011.2118370

[2] H. Fattahi, H.G. Barros, M. Gorjan, T. Nubbemeyer, B. Alsaif, C.Y. Teisset, M. Schultze, S. Prinz, M. Haefner, M. Ueffing, et al., Third-generation femtosecond technology, Optica 1, 45-63 (2014), https://doi.org/10.1364/OPTICA.1.000045

[3] F. Batysta, R. Antipenkov, J. Novák, J.T. Green, J.A. Naylon, J. Horáček, M. Horáček, Z. Hubka, R. Boge, T. Mazanec, B. Himmel, P. Bakule, and B. Rus, Broadband OPCPA system with $11 \mathrm{~mJ}$ output at $1 \mathrm{kHz}$, compressible to $12 \mathrm{fs}$, Opt. Express 24, 17843-17848 (2016), https://doi. org/10.1364/OE.24.017843 
[4] J. Liu, W. Wang, Z. Wang, Z. Lv, Z. Zhang, and $Z$. Wei, Diode-pumped high energy and high average power all-solid-state picosecond amplifier systems, Appl. Sci. 5, 1590-1602 (2015), https://doi.org/10.3390/app5041590

[5]J. Adamonis, A. Aleknavičius, K. Michailovas, S. Balickas, V. Petrauskiene, T. Gertus, and A. Michailovas, Implementation of a SVWPbased laser beam shaping technique for generation of 100-mJ-level picosecond pulses, Appl. Opt. 55, 8007-8015 (2016), https://doi.org/10.1364 AO.55.008007

[6] M.Y. Shverdin, F. Albert, S.G. Anderson, S.M. Betts, D.J. Gibson, M.J. Messerly, F.V. Hartemann, C.W. Siders, and C.P.J. Barty, Chirpedpulse amplification with narrowband pulses, Opt. Lett. 35(14), 2478-2480 (2010), https://doi. org/10.1364/OL.35.002478

[7] L. Veselis, R. Danilevičius, A. Zaukevičius, A. Michailovas, and N. Rusteika, OPCPA pump source based on chirped second harmonic pulse compression, in: Proceedings of the 7th EPS-QEOD Europhoton Conference on Solid State, Fibre, and Waveguide Coherent Light Sources (European Physical Society, 2016).

[8] K. Michailovas, V. Smilgevicius, A. Michailovas, and A. Zaukevicius, Neodymium doped active medium based high power high energy 10-20 ps pulse amplification system using chirped pulse amplification technique, in: Advanced Solid State Lasers, OSA Technical Digest (online), paper ATh2A.27 (Optical Society of America, 2014), https://doi.org/10.1364/ASSL.2014.Ath2A.27

[9] K. Michailovas, A. Baltuska, A. Pugzlys, V. Smilgevicius, A. Michailovas, A. Zaukevicius, R. Danilevicius, S. Frankinas, and N. Rusteika, Combined $\mathrm{Yb} / \mathrm{Nd}$ driver for optical parametric chirped pulse amplifiers, Opt. Express 24, 2226122271 (2016), https://doi.org/10.1364/OE.24.022261

[10]L.M. Frantz and J.S. Nodvik, Theory of pulse propagation in a laser amplifier, J. Appl. Phys. 34, 23462349 (1963), https://doi.org/10.1063/1.1702744

[11]M.P. Kalashnikov, K. Osvay, I.M. Lachko, H. Schonnagel, and W. Sandner, Broadband amplification of $800-\mathrm{nm}$ pulses with a combination of negatively and positively chirped pulse amplification, IEEE J. Sel. Top. Quant. Electron. 12, 194-200 (2006), https://doi.org/10.1109 ISTQE.2006.872730

[12]F. Giambruno, C. Radier, G. Rey, and G. Chériaux, Design of a $10 \mathrm{PW}(150 \mathrm{~J} / 15 \mathrm{fs})$ peak power laser system with Ti:sapphire medium through spectral control, Appl. Optics 50, 2617-2621 (2011), https://doi.org/10.1364/AO.50.002617

[13]D. Schimpf, J. Limpert, and A. Tünnermann, Optimization of high performance ultrafast fiber laser systems to $>10 \mathrm{GW}$ peak power, J. Opt. Soc. Am. B 27, 2051-2060 (2010), https://doi. org/10.1364/JOSAB.27.002051

[14]Y. Sato and T. Taira, Temperature dependencies of stimulated emission cross section for Nd-doped solid-state laser materials, Opt. Mater. Express 2, 1076-1087 (2012), https://doi.org/10.1364/ OME.2.001076

[15]W. Koechner, Solid-State Laser Engineering (Springer-Verlag New York, 2006).

[16]C. Bibeau, S.A. Payne, and H.T. Powell, Direct measurements of the terminal laser level lifetime in neodymium-doped crystals and glasses, J. Opt. Soc. Am. B 12, 1981-1992 (1995), https://doi. org/10.1364/JOSAB.12.001981

[17]D. Auric and A. Labadens, On the use of a circulary polarized beam to reduce the self-focussing effect in a glass rod amplifier, Opt. Commun. 21, 241-242 (1977), https://doi.org/10.1016/00304018(77)90272-3

[18]M.D. Perry, R.D. Boyd, J.A. Britten, D. Decker, B.W. Shore, C. Shannon, and E. Shults, Highefficiency multilayer dielectric diffraction gratings, Opt. Lett. 20, 940-942 (1996), https://doi. org/10.1364/OL.20.000940

[19]]B.W. Shore, M.D. Perry, J.A. Britten, R.D. Boyd, M.D. Feit, H.T. Nguyen, R. Chow, G.E. Loomis, and L. Li, Design of high-efficiency dielectric reflection gratings, J. Opt. Soc. Am. A 14, 1124-1136 (1997), https://doi.org/10.1364 JOSAA.14.001124

[20]K. Hehl, J. Bischoff, U. Mohaupt, M. Palme, B. Schnabel, L. Wenke, R. Bödefeld, W. Theobald, E. Welsch, R. Sauerbrey, and H. Heyer, Highefficiency dielectric reflection gratings: design, fabrication, and analysis, Appl. Opt. 38, 
6257-6271 (1999), https://doi.org/10.1364 AO.38.006257

[21]F. Kong, Y. Jin, H. Huang, H. Zhang, S. Liu, and H. He, Laser-induced damage of multilayer dielectric gratings with picosecond laser pulses under vacuum and air, Opt. Laser Technol. 73,
39-43 (2015), https://doi.org/10.1016/j.optlastec. 2015.03.011

[22]R. Paschotta, The Encyclopedia of Laser Physics and Technology, https://www.rp-photonics.com. accessed: 09/08/2017.

\title{
IKI 20 ps TRUKMĖS DIDELE்S ENERGIJOS IMPULSAI IŠ 1 kHz ČIRPUOTŲ IMPULSŲ STIPRINTUVO Nd JONAIS LEGIRUOTŲ AKTYVIŲJŲ TERPIỤ PAGRINDU
}

\author{
K. Michailovas a, , A. Zaukevičius a, c, V. Petrauskienè ${ }^{\text {a }}$, V. Smilgevičius ${ }^{\text {b }}$, \\ S. Balickas ${ }^{a}$, A. Michailovas ${ }^{\text {a, }} \mathrm{c}$ \\ ${ }^{\text {a }}$ EKSPLA, Vilnius, Lietuva \\ ${ }^{\mathrm{b}}$ Vilniaus universiteto Lazeriniu tyrimu centras, Vilnius, Lietuva \\ ${ }^{\mathrm{c}}$ Fiziniu ir technologijos mokslu centro Fizikos institutas, Vilnius, Lietuva
}

\section{Santrauka}

Naudojant čirpuotų impulsų stiprinimo (angl. CPA) metodą, sukurti $\mathrm{Nd}: \mathrm{YAG}$ ir $\mathrm{Nd}: \mathrm{YVO}_{4}$ stiprintuvai. Skaidulinis osciliatorius pagamintas $\mathrm{Yb}$ jonų legiruotos skaidulos pagrindu kartu su čirpuota skaiduline Braggo gardele, kuri kaip plèstuvas suformuodavo užkratą tolimesniam stiprinimui kieto kūno $\mathrm{Nd}$ jonais legiruotu stiprintuvu grandineje. Sustiprinti impulsai buvo spaudžiami gardeliniame spaustuve, surinktame iš specialiu didelès dispersijos daugiasluoksnių dielektrinių difrakcinių gardelių. Tai leido sukurti santykinai kompaktišką ir vidutinio sudètingumo osciliatoriausstiprintuvo sistemą, kurios išejime mes gavome $85 \mathrm{~mJ}$ energijos ir mažesnès nei 20 ps trukmès impulsus $1 \mathrm{kHz}$ pasikartojimo dažniu. Šie stiprinimo sistemos parametrai yra itin tinkami optinio parametrinio čirpuotų impulsų stiprintuvo (angl. OPCPA) kaupinimui. 